\title{
A LOW COST SHORT RANGE WIRELESS EMBEDDED SYSTEM FOR MULTIPLE PARAMETER CONTROL
}

\author{
Sudhindra $\mathrm{F}^{1}$, Annarao. S. $\mathrm{J}^{2}$, Vani R. $\mathbf{M}^{3}$, P.V. Hungund ${ }^{4}$ \\ ${ }^{I}$ Technical Officer-I, U.S.I.C, Gulbarga University, Gulbarga, Karnataka, India \\ ${ }^{2}$ Head, Dept. of Electronics, HKE's Women's Polytechnic, Gulbarga, Karnataka, India \\ ${ }^{3}$ Head, USIC, Gulbarga University, Gulbarga, Karnataka, India \\ ${ }^{4}$ Professor, Dept. of Applied Electronics, Gulbarga University, Gulbarga, Karnataka, India
}

\begin{abstract}
It is well established fact that the process atomization offers the advantages like high accuracy, power saving, manpower saving, reduction in wastage, high \& efficient production volumes. In the modern industries precise monitoring, \& controlling of temperatures \& fluid level of various chemicals in storage tanks at various places is an essential requirement. This paper describes the development of Wireless Embedded System by using Atmel's 89C51 microcontroller for monitoring \& control of process parameters from remote site .The system utilizes ASK transmitter \& receiver for transmission and reception of reference values i.e., temperature and fluid levels from transmitter to receiver. Interaction with transmitter is done through matrix keypad. A TRIAC AC power controller circuit is used in the receiver which controls the flow of power to the heater. The fluid level is maintained by a water feed pump. User friendly Software is developed using 8051 's Assembly language to control the transmitter and receiver units.
\end{abstract}

Keywords: ASK/RF transceiver, Temperature sensor, Triac, Fluid level sensor and Microcontroller etc...

\section{INTRODUCTION}

Microcontrollers have been used in the recent past in various industrial applications and R \& D for controlling and monitoring various parameters. Automated monitoring and controlling of various process parameters, through the use of electronic techniques are in use since a long time. Such systems have become essential and it has always yielded better results over their manual counterparts. However such systems suffer from some disadvantages like the overshoots and undershoot in the controlled parameters, since they use relay type control and they allow the monitoring and control of process parameters only from close vicinity. Besides to adjust set points and periodic recording of parameters, an operator is required. Microcontroller applications in dedicated system assumed an important place in engineering, especially the large scale industries.

With the advancement in techniques for control systems and additional requirement of miniaturization, microcontrollers have become the most suitable components. We find such application [1], in which microcontroller based temperature indicator \& controller was developed which can be used in process industry for monitoring \& control of temperature. In the other application A. Rajendran et al, have developed a data acquisition system with AT89C52 microcontroller \& PID algorithm [2].
In another work J. Jayapandian et al, has developed single chip embedded temperature controller and they have used Programmable System on Single Chip (PSOC). The LabVIEW is used to implement the control program.[3],

Our proposed work aims at designing a wireless embedded system for multiple parameter monitoring and control using thyristors and microcontrollers. An attempt is made to design hardware and software for a compact, reliable, and low cost system to achieve remote process automation. In this system process automation is implemented for the temperature and fluid level measurement. However, any other physical parameters like pressure, flow, illumination, DC motor speed, AC motor speed, conveyer belt speed etc can also be easily implemented.

\section{SYSTEM DESCRIPTION}

The block diagram of the Wireless Embedded System for multiple parameter control is shown fig.1 and fig. 2. The system hardware consists of two sections i.e. Transmitter Unit (TU) and Receiver Unit (RU).

The Transmitter Unit consists of an AT89C51 microcontroller, 4X4 matrix keyboard, 16 X 2 LCD display unit, ASK/RF transmitter, ASK/RF receiver, buzzer. The microcontroller continuously scans the keypad and checks for the key pressings. If any key is pressed then it sends the information to LCD display unit and to the SBUF register which will be 
transmitted through the TxD line of the serial port. The data will be converted into ASK tones by ASK modulator which is then converted into electromagnetic waves by an antenna and transmitted to open space at $433 \mathrm{MHz}$ frequency (license free ISM Band).

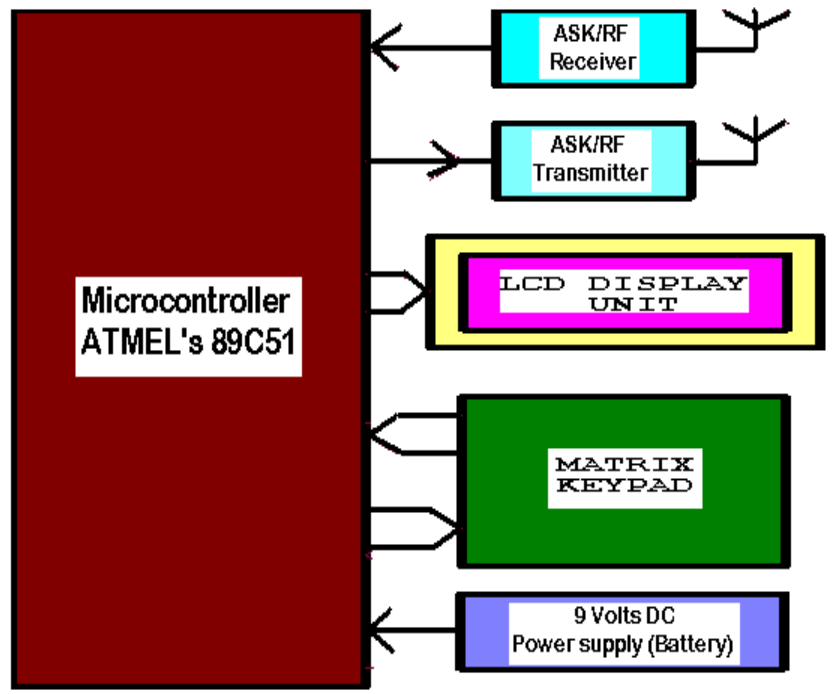

Fig-1: Block diagram of Transmitter Unit

The Receiver Unit consists of an AT89C51 microcontroller, $\mathrm{ASK} / \mathrm{RF}$ receiver, $\mathrm{LCD}$ display unit, analog to digital converter (ADC), digital to analog converter (DAC), TRIAC $\mathrm{AC}$ power controller circuit and optical isolator, temperature \& level sensors, signal conditioner, pump \& pump driver relay and a container attached with heater.

The receiver intercepts electromagnetic waves in the $433 \mathrm{MHz}$ band by the receiving antenna and recovers the ASK tones after demodulation to obtain the original digital data. The microcontroller processes these data and utilizes it to display the set point values. Then it reads present values of both parameters through ADC, compares both values and changes the firing angle in order to adjust present values equal to set point . The DAC is used to adjust the conduction period of the TRIAC by controlling the PWM signal. The binary value sent to the DAC is dynamically adjusted by the microcontroller corresponding to the error signal. The microcontroller in RU displays the present parameter values, conduction angle and other status information locally and also sends this in the form of electromagnetic waves towards TU. The TU recovers this information and displays it in real time.

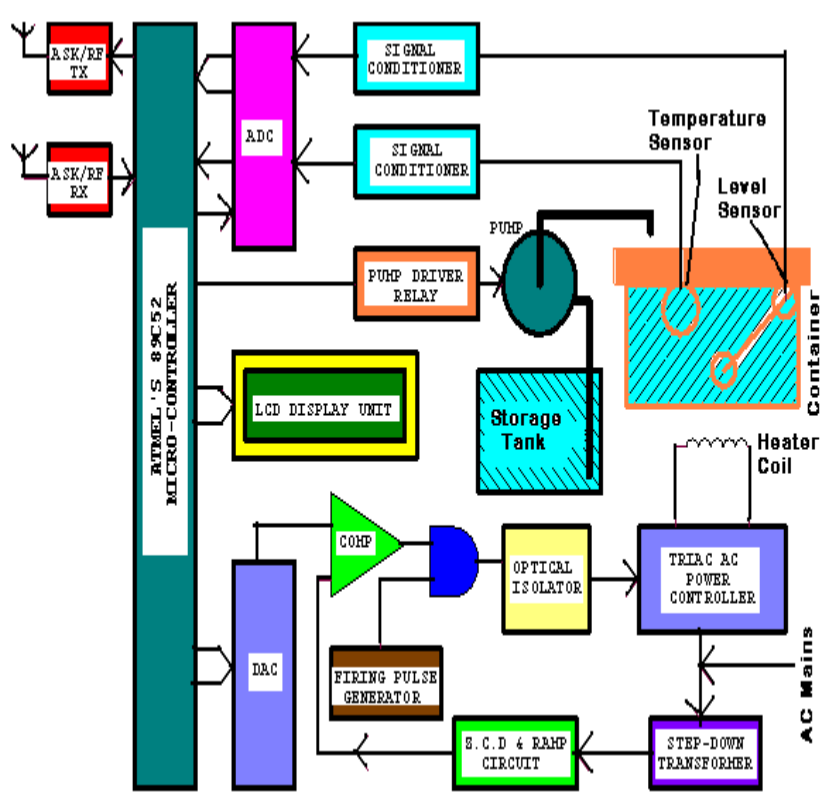

Fig-2: Block diagram of Receiver Unit

\section{HARDWARE FEATURES}

The different blocks of the system are discussed in this section.

\subsection{Microcontroller}

Microcontrollers are the most important part of this system. They perform measurement and control functions. This scheme uses two Atmel's AT89C51microcontrollers. The microcontroller in the Transmitter Unit controls Matrix Keypad, ASK/RF transmitter/receiver, and LCD display. The microcontroller in the Receiver Unit controls the ADC, DAC, LCD display ASK/RF Receiver/transmitter and relay.

\subsection{Matrix Keypad}

The matrix keypad is used by the operator to interact with the system. It is a $4 \times 4$ matrix hex keypad designed using 16 soft push button keys.

\subsection{ASK/RF Transmitter}

This unit modulates the serial data from the microcontroller with a high frequency carrier signal in the license free ISM band of $433 \mathrm{MHz}$ to produce ASK signals and then radiates them into the free space in the form of electromagnetic waves with the help of a short antenna and it is used both in TU \& RU sections.

\subsection{ASK/RF Receiver}

The receiver intercepts the electromagnetic waves from the free space. Then selects the $433 \mathrm{MHz}$ signals and demodulates them with its built-in demodulator to get the original serial 
data and then sends it to the microcontroller on serial input line. The microcontroller reconstructs and identifies the code and then takes action to control the devices. This receiver is used both in TU \& RU sections.

\subsection{LCD Display}

LCD module is used as the main output unit for displaying process parameter values. It displays the Present temperature value and Set point temperature values. It also displays the present value of firing angle. A 2-line, 16 Character type LCD modules with backlit facility is used in this work. The microcontroller sends the signals to LCD module through its ports and is used in both TU \& RU sections.

\subsection{Buzzer Driver Circuit}

Buzzer is used to issue warning signal to the operator if the parameters are above the threshold values. It uses a peizoelectric buzzer and a driver transistor.

\subsection{Digital to Analog Converter (DAC)}

Digital to analog converter (DAC-0800) is used to convert 8bit binary number sent by the microcontroller into an analog DC voltage. The microcontroller sends an appropriate binary count to DAC to set the required firing angle. This DC voltage is fed to the inverting input of comparator to adjust the firing angle. The firing angle increases as the DC voltage from the DAC increases and the firing angle decreases as the DC voltage from DAC decreases. The power flow to the heater varies with the firing angle.

\subsection{Comparator}

The comparator produces a Pulse Width Modulated (PWM) signal, which controls the firing angle of TRIAC to control the amount of load current. The comparator circuit receives the ramp signal as one input and variable DC voltage produced from the DAC as another input. The comparator's output remains low as long as the ramp amplitude is below the DC voltage level. It becomes high when the ramp amplitude crosses the DC voltage level. This variation in the DC voltage changes the firing angle and permits the AND gate to pass firing pulses to TRIAC to turn on the heater.

\subsection{Firing Pulse Generator}

It is an astable-multivibrator built using NE555 timer IC. It produces high frequency square wave pulses at TTL level. These pulses are used to fire the thyristors. Pulse Width Modulated (PWM) signal generated by a comparator and ramp generator controls these firing pulses.

\subsection{Zero-Cross-Detector (ZCD)}

Since phase control technique is used in order to supply only required amount of power to the load. The microcontroller requires $\mathrm{ZCD}$ pulses to synchronize with $\mathrm{AC}$ mains. Zerocross-detector circuit is used to provide reference pulses to synchronize with every half cycle of AC mains voltage. The differentiated pulses are fed to a ramp generator. It uses LM324 Op-Amp.

\subsection{Ramp Generator}

Ramp generator circuit is used to produce a ramp signal, which is synchronized with A.C mains. The ramp is used to fix the required firing angle. It uses a transistor to produce a ramp signal and the amplitude of this ramp is adjusted to 10 Volts. The ramp signal from this stage is fed to non-inverting input of the comparator. The inverting input of the comparator receives a D.C voltage produced by DAC. Ramp signal is compared with the D.C voltage, and a PWM signal is generated at the output of the comparator. The duty cycle of the PWM signal is adjusted to provide required firing and conduction angles.

\subsection{Triac}

The TRIAC BT136 is used to control the flow of AC power to the heater. The conduction of TRIAC in each half cycle starts when it receives a firing pulse at it's gate. The conduction continues till the end of that half cycle.. The microcontroller controls the firing pulse which in turn controls the power delivered to the heater according to the difference between reference values and actual values. A RC snubber circuit is provided to protect the TRIAC against transient.

\subsection{Temperature Sensor}

The temperature sensor chosen used here is LM35D. It is an industry standard semiconductor temperature transducer suitable for the temperature range of $-50^{\circ} \mathrm{C}$ to $+150^{\circ} \mathrm{C}$. It is a voltage $\mathrm{o} / \mathrm{p}$ device. It produces a DC voltage proportional to surrounding temperature. This voltage is internally calibrated to provide $10 \mathrm{mVolts} /$ degree centigrade.

\subsection{Fluid Level Sensor}

The fluid level sensor is used to sense the current fluid level in the storage tank. It is basically a potentiometer with a floating ball attached to its spindle with a lever mechanism. The floating ball always floats over the fluid surface and rotates the potentiometer in either direction as the fluid level changes. The part of the DC excitation voltage applied across the potentiometer is available at the centre terminal of the potentiometer. This provides a voltage proportional to the fluid level in the tank. This voltage is an analog signal and hence it is converted into a digital value by using an ADC.

\subsection{Signal Conditioner}

LM 324 Operational Amplifier is used as signal conditioner. It provides the required gain to the temperature signal before it 
is fed into the ADC. It also facilitates for the calibration of the sensor output signal.

\subsection{Analog to Digital Converter}

The analog to digital converter converts the analog signal into an equivalent digital value. The analog values of temperature and fluid levels are fed to ADC converter to get digital values. The microcontroller reads the digital data from ADC. An 8bit, successive approximation type ADC 0809 is used in this system.

\subsection{Fluid Container and Pump Relay}

The fluid container is attached with heater, pump outlet, temperature and fluid level sensors. The pump is controlled by an electromagnetic relay. The microcontroller controls this relay in order to turn $\mathrm{ON}$ or OFF the pump motor.

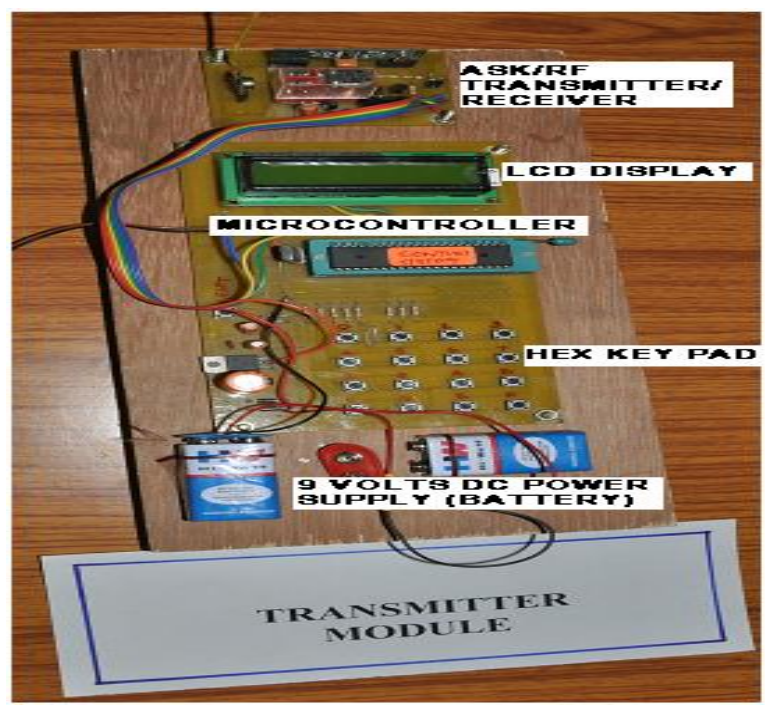

Fig.3: Photograph of Wireless Transmitter Unit

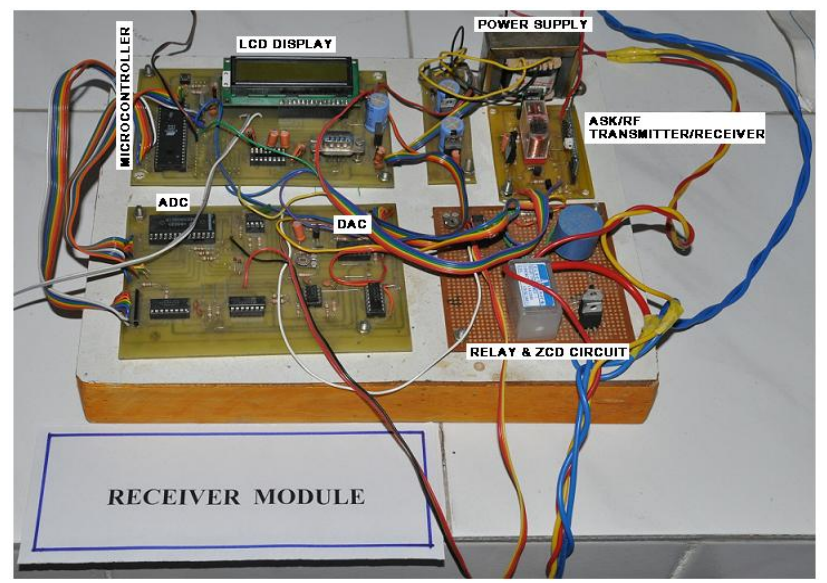

Fig.4: Photograph of Wireless Receiver Unit

\section{SOFTWARE FEATURES}

User friendly software is written in 8051 assembly language which will ensure the compact code size. The program in the microcontroller of transmitter unit initializes the serial ports, LCD display unit, buzzer and scans the matrix keypad and displays an initial message on the LCD display. Then it waits for the operator to enter reference values through the keypad. When the operator enters the reference values for both parameters, it displays these values locally on the LCD unit, and also sends it to SBUF register of the serial port from where it is sent serially to ASK transmitter. Then it waits for the data pockets to be received on its serial port from the Receiver unit. Once the packets of data from the receiver terminal are received, it decodes it and recovers the present values of both parameters and displays them on LCD. If the present values exceed the reference values it sounds the buzzer.

The receiver unit microcontroller is programmed to initialize the LCD, ADC, DAC, buzzer and pump relay. It waits for the reference values to be received by its serial port through ASK receiver. When packets of data are received, it decodes and recovers the reference values, stores and displays them on LCD. Then it activates ADC to obtain current values of parameters and compares them with their respective reference values and changes the firing angle in order to adjust the current values to reference values. The DAC then changes the conduction period of triac by controlling the PWM signal. The binary values sent to the DAC are dynamically adjusted by the microcontroller corresponding to the error signals. Mean while it sends the present values to the serial port from there they are sent to ASK transmitter and this will be received by the transmitter unit. The microcontroller does the above operations through the program stored in it.

\section{EXPERIMENTAL RESULTS \& DISCUSSIONS}

The designed work mainly concentrates on atomization, remote processing, power saving. The real time display of current values of the physical parameters from receiver unit to transmitter unit constantly and which leads to minimization of human interference. The individual system components as well as the integrated units are thoroughly tested in the laboratory for different reference values of physical parameters i.e. temperature and $\%$ of fluid level.

The two graphs shown in fig. 5 \& fig.6 are the data from the RU. The fig. 3 is the graph of temperature versus time. Here the exponential portion of the curve indicates that the temperature starts rising from initial value i.e. $28 \mathrm{deg}$. $\mathrm{C}$ to reference value i.e. 50 deg. C. When it reaches to reference value, slowly the power input to heater is reduced and the heater comes to off position. The fig. 4 shows the graph for $\%$ of fluid level versus time in the storage tank. The motor is made on for initial value of fluid level \& is kept ON during the exponential portion of the curve. When the fluid level reaches 
to reference value (here it is $60 \%$ of the container), the relay turns off the pump motor.

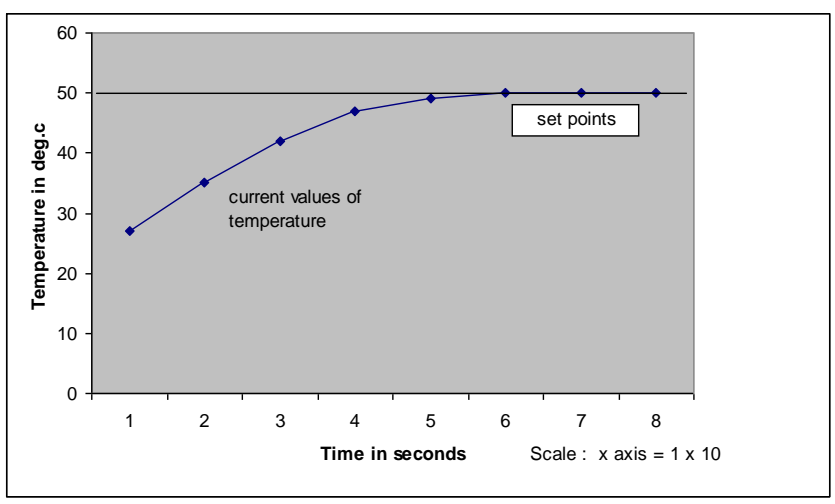

Fig-5: Temperature VS Time in Sec

It has been observed that the designed system works properly as per the control logic. From the above discussions it is clear that the system is automatic, wireless, real time monitoring and does the control of parameters within the range of $300 \mathrm{ft}$ even in the hazard industrial environment.

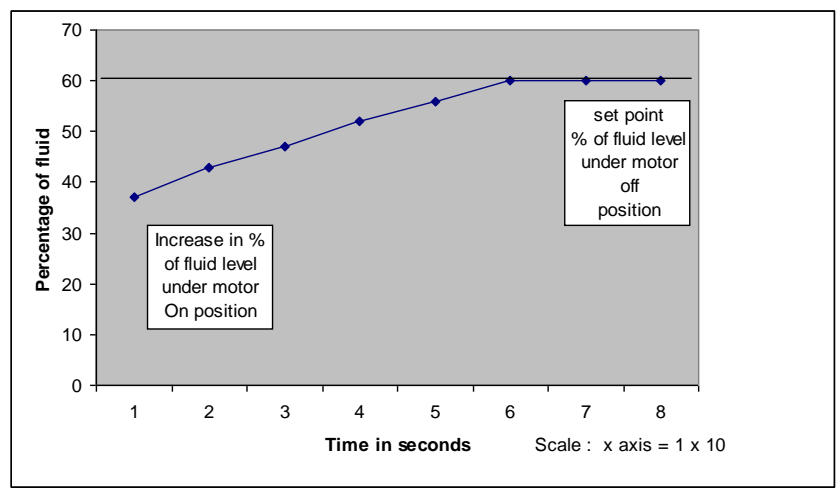

Fig-6: \% of Fluid VS Time in Sec

\section{CONCLUSIONS}

The wireless embedded system for multiple parameter control using Atmel's 89C51 microcontroller is designed and developed successfully in the laboratory. The developed system is simple, low cost, and suitable for multiple process parameter control. This design permits the user to adjust process parameters from the remote transmitter unit. As it is a real time system it will display both reference and current values of the parameters on the LCD of transmitter continuously. The developed system also issues warning signals to the operator within the vicinity of transmitter so that he can interact with the system. The system is wireless and the distance between TU \& RU can be up to $300 \mathrm{ft}$. The system with minor modifications can be used for various types of process parameter control and suitable for Industrial applications where remote control is necessary.

\section{REFERENCES}

[1]. R.G. Jamkar and R.H. Chile "Microcontroller based Temperature Indicator and Controller", J. Instrum. Soc . India 34(3) 180-186, Sept-2004.

[2]. A.Rajendran and P. Neelamegam "Design of AT89C52 Microcontroller based system for the measurement of Temperature and control", J. Instrum. Soc , India 35(I)99-105, March-2005.

[3]. J. Jayapandian and Usha Rani Ravi "An Embedded Single Chip Temperature Controller Design" J. Instrum, Soc. India 38(2) 132-136, June-2008.

[4]. Kenneth J. Ayala, "The 8051 Microcontroller Architecture , Programming and Applications", 2nd Edition , Penaram International Publishing (India), 1996.

[5]. Ramakant Gaikwad, "Op-amps and linear integrated circuits", 3rd Edition Prentice- Hall of India Pvt. Ltd, New Delhi.

[6]. Muhammad Ali Mazidi, Janice Gillispie and Rolin D. McKinlay "The 8051 Microcontroller and Embedded Systems using Assembly and 'C'., Prentice - Hall of India, New Delhi, 2nd Edition .

\section{BIOGRAPHIES}

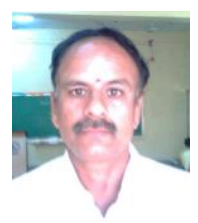

Sudhindra F. Received his B.E (E\&CE) from Gulbarga University, Gulbarga and Master's degree from KSOU, Mysore. He is working as Technical Officer-I in University Science Instrumentation Centre, Gulbarga University, Gulbarga since 1995. He has completed his M.Phil from Gulbarga University, Gulbarga. He has attended many seminars, workshops and conferences. His areas of interest are digital electronics, Embedded Controllers and Wireless communication. He has serviced and repaired more than 500 laboratory instruments.

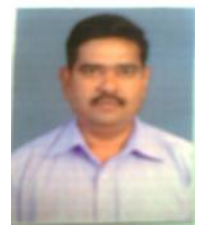

Annarao.S.J. received his B.Tech (E\&CE) from Guru Ghasidas University, Bilaspur Chattisgarh and pursuing Master's degree from KSOU, Mysore. He is working as Head of Department in Electronics and Communication Engineering Department of HKE Society's women's polytechnic, Gulbarga. He has attended many seminars, workshops and conferences. His areas of interest are embedded systems, Biomedical, RFID, GSM, GPS, Wireless communication and Computers. He has guided more than 500 live projects for diploma, BE and M.Tech students.

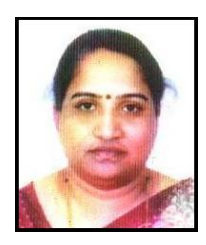

Vani R. M. received her B.E. in Electrical and Electronics from the B.I.ET., Davanagere and M.Tech in Industrial Electronics from S.J.C.E., Mysore, Karnataka. She has received her Ph.D in Applied Electronics from Gulbarga University, Gulbarga, India, in year 
2005. She is working as Reader \& Head, University Science Instrumentation Center, Gulbarga University, Gulbarga, since 1995. She has more than 85 research publications in national and international reputed journals/Conference proceedings. she presented the research papers in National/ International conferences in India and abroad. She has conducted several courses, workshops for the benefits of faculties and field engineers. Her areas of interest are microwave antennas, PC based instrumentation, embedded controllers and Wireless communication. She has one UGC major research project to her credit

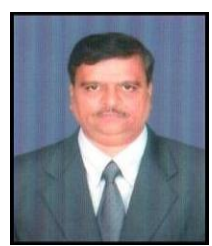

P. V. Hunagund, received his M.Sc and Ph.D from the Dept. of Applied electronics, Gulbarga University, Gulbarga, in the year 1982 and 1992 respectively. He is working as professor and chairman of Applied Electronics department, Gulbarga University, Gulbarga. He has more than 100 research publications in national and international reputed journals, more than 150 research publications in international symposium/Conferences. $\mathrm{He}$ has presented the research papers in National/International conferences in India and abroad. He has guided many Ph.D and M.Phil students. He has three major research projects at his credit. He has worked as a committee member in various selection committees for selection of Associate Professor and Professor in different Universities. 